\title{
MATHEMATICAL MODEL OF DIESEL ENGINE CHARACTERISTICS FOR DETERMINING THE PERFORMANCE OF TRACTION DYNAMICS OF WHEEL-TYPE TRACTOR
}

\author{
Oleksii Rebrov \\ Department of Car and Tractor Industry ${ }^{1}$ \\ alexrebrov0108@gmail.com \\ Andrii Kozhushko \\ Department of Car and Tractor Industry ${ }^{1}$ \\ andreykozhushko7@gmail.com \\ Boris Kalchenko \\ Department of Car and Tractor Industry ${ }^{1}$ \\ kabor78@ukr.net \\ Anatoliy Mamontov \\ Department of Car and Tractor Industry ${ }^{1}$ \\ monkhoktar@gmail.com \\ Alexander Zakovorotniy \\ Department of Computer Engineering and Programming 1 \\ zakovorotniy@kpi.kharkov.ua \\ Evgeniy Kalinin \\ Department of Reliability, Durability and Technical Service of Machines \\ Kharkiv Petro Vasylenko National Technical University of Agriculture \\ 44 Alchevskykh str., Kharkiv, Ukraine, 61002 \\ kalininhntusg@gmail.com \\ Elena Holovina \\ Department of Automobile Transport and Transport Technology \\ Filial of Classic Private University \\ 24/37 Heavenly hundreds str., Kremenchuk, Ukraine, 39600 \\ elenholz@gmail.com \\ ${ }^{1}$ National Technical University "Kharkiv Polytechnic Institute" \\ 2 Kyrpychova str., Kharkiv, Ukraine, 61002
}

\footnotetext{
Abstract

Wheel-type tractors carry out a range of processing operations, with the exception of early spring work, when caterpillar tractors are used to reduce the compaction effect on the soil. Therefore, to plan the costs and reserves associated with fuel consumption, it is necessary to have an estimate of the fuel economy of the tractor in basic agricultural operations.

An objective assessment of fuel consumption requires a mathematical model that describes the fuel characteristics of the engine, taking into account the speed and load torque in a wide range of variation. Verification of the model is possible only with experimental data.

Since the efficiency and fuel economy of a tractor depends not only on engine performance, but also on the perfection of the transmission, the running system and the rational choice of speed, it is necessary to take into account the time-varying nature of the tractor's traction load. The complex of agricultural operations can be divided into characteristic cycles of load change over time. This principle is the basis of PowerMix test cycles, which are conducted on a concrete track to ensure repeatability of the experiment. The use of the variable load on the tractor in the PowerMix tests is positive, but in actual field tests the results may differ due to the
} 
instability of the soil properties. On the other hand, PowerMix field cycles can be taken as standard test loads in the simulation of tractor traction tests on the ground.

Keywords: diesel engine, specific fuel consumption, approximation of specific fuel consumption, fuel efficiency, PowerMix test cycles.

DOI: $10.21303 / 2461-4262.2020 .001352$

\section{Introduction}

To assess fuel economy and determine hourly fuel consumption in partial engine operation modes, a model [1-3] is used that takes into account the crankshaft rotational speed and engine load in terms of power relative to the rated power taken through the tractor power take-off shaft. The coefficients of this model are determined by the results of tests on five engine operation modes. The peculiarity of the models hourly fuel consumption [1-3] is the use of experimental data of three modes of engine operation with full throttle supply and only two modes with incomplete fuel supply. That is, fuel consumption in partial modes is modeled using two points. Also, the hourly fuel consumption model [1-3] does not take into account the nature of the external speed characteristics of the engine. In addition, when building it, fuel consumption data are not used at a crankshaft rotational speed lower than at maximum torque, because it is in this mode range that the diesel engine is highly economical [4]. Therefore, the use of the model [1-3] for simulation of tractor traction dynamics is difficult. It should also be noted that the type of transmission of the tractor and the mode of its operation significantly affect the fuel economy. So in [5], a significant difference is established in the hourly fuel consumption during automatic and manual operation of a continuously variable transmission. A similar difference is found in [6] when comparing a stepwise and continuously variable transmission, as well as a reception, consists in shifting to a higher gear while "shift up and throttle back" (SUTB). Hourly fuel consumption can be estimated according to test reports of the German Agricultural Society (DLG, Deutsche Landwirtschafts-Gesellschaft) according to the PowerMix procedure [7], or the testing laboratory of the University of Nebraska in the USA (University of Nebraska, Lincoln, Nebraska tractor test laboratory) [8]. The test procedure is carried out according to the OECD Code 2 protocol [9]. The hourly fuel consumption model [1-3] is based precisely on the test reports of the University of Nebraska [8]. But the use of DLG protocols [7] makes it possible to use the data of experimental tests in four partial modes when constructing a fuel consumption model, instead of two of the protocols [8].

Therefore, the aim of the work is to develop a model of the characteristics of a diesel engine and fuel consumption to assess the fuel economy of a tractor when modeling with PowerMix field cycles on the ground.

To achieve the intended aim, it is necessary to complete the following objectives:

- to formulate a methodology for approximating the fuel characteristics of the engine using the experimental data of the external speed characteristics and partial engine operation modes;

- to develop a mathematical model of the characteristics of a diesel engine to determine the traction dynamics of a wheel-type tractor and to test it in a simulation with PowerMix field cycles.

\section{Mathematical modeling of diesel engine characteristics}

To assess the energy performance and fuel efficiency of the engine in the entire load and speed range of operating modes, it is convenient to use a universal or multi-parameter characteristic, according to which the specific effective fuel consumption is determined by the expression [10]:

$$
g_{e}=g_{n} \cdot k_{N} \cdot k_{\omega}
$$

where $k_{N}, k_{\omega}$ - coefficients that take into account the engine load in terms of power and speed; $g_{n}-$ specific effective fuel consumption of the engine in nominal mode.

The coefficients $k_{N}, k_{\omega}$ are usually approximated by polynomials, [10]:

$$
k_{\omega}=a_{\omega}+b_{\omega} \cdot \varepsilon_{\omega}+c_{\omega} \cdot \varepsilon_{\omega}^{2}
$$




$$
\begin{gathered}
a_{\omega}+b_{\omega}+c_{\omega}=1 ; \\
k_{N}=a_{N}+b_{N} \cdot \varepsilon_{N e}+c_{N} \cdot \varepsilon_{N e}^{2} \\
a_{N}+b_{N}+c_{N}=1
\end{gathered}
$$

where $\varepsilon_{\omega}$ - ratio of reduced- and full-throttle engine speeds at operating load (decimal); $\varepsilon_{N e}-$ ratio of reduced- and full-throttle engine power at operating load (decimal):

$$
\varepsilon_{N e}=\frac{N_{e}}{N_{e P}},
$$

where $N_{\mathrm{e}}, N_{\mathrm{eP}}$ - effective engine power in the current mode and full-throttle engine power at the same speed mode.

In the presence of experimental data, the engine performance model can be substantially refined. At the first stage, it is necessary to approximate full-throttle engine performance characteristic. The most universal method is spline approximation, which allows one to obtain a functional dependence of the specific fuel consumption with a confidence $R^{2}=1$ :

$$
\frac{g_{e}}{g_{n}}=k_{\omega}=f_{g e}\left(\varepsilon_{\omega}\right)
$$

Replacing (2) with (5) significantly improves the quality of approximation of the specific fuel consumption rate by engine speed. The next step of refinement may be to use the load characteristics of the engine or their series. In their absence, an alternative may be the DLG test protocols according to the PowerMix procedure [7], which, in terms of engine testing, is carried out according to the OECD Code 2 protocol [9], which assumes measurements of fuel consumption in partial modes when loading at a speed $\varepsilon_{\omega}$ and power $\varepsilon_{N}$ according to the Table 1.

Table 1

Partial engine test modes according to the OECD Code 2 protocol [9]

\begin{tabular}{ccc}
\hline Measurement point & $\boldsymbol{\varepsilon}_{\boldsymbol{\omega}}$ & $\boldsymbol{\varepsilon}_{\mathbf{N}}$ \\
\hline$\# 1$ & $>1$ & 0,8 \\
$\# 2$ & 0,9 & 0,8 \\
$\# 3$ & 0,9 & 0,4 \\
$\# 4$ & 0,6 & 0,6 \\
$\# 5$ & 0,6 & 0,4
\end{tabular}

The data in Table 1 allow the polynomial (3) to be approximated with respect to the load $\varepsilon_{N}$ at $\varepsilon_{\omega}=$ const, according to modes 2-3 or 4-5. It is correct to use these 4-5 modes, since they are closer to the nominal one, which will reduce the discrepancy between the theoretical calculation and the experiment. Also, in accordance with the diesel engine operating model of wheel-type agricultural tractors, engines $70 \%$ of the time of the annual employment cycle are operated at $\varepsilon_{\omega}>0.9$ (with a share of the minimum idle mode of $15.4 \%$ ). When approximating (3), the second-degree polynomial of the third point is the regime of full-throttle characteristic determined by the previously approximated dependence (5) $\varepsilon_{\omega}=0.9$. Moreover, the mandatory fulfillment of condition (3) is also necessary, so that the reliability of the approximation $R^{2} \neq 1$ can't always be fulfilled.

After approximating the surface of fuel consumption (1), the calculation results are refined in accordance with the data of fuel consumption in the modes of the Table 1, and in the 
regimes of the regulatory branch of the full-throttle characteristic using the probability density function of the two-dimensional normal distribution. In this case, the mathematical expectation vectors of the angular velocity of the engine crankshaft $M_{\omega}$, the effective torque $M_{M}$, the effective power $M_{N}$ in the operating modes according to the Table $\mathbf{1}$ are formed and on the regulatory branch of the full-throttle characteristic and experimentally determined fuel consumption $g_{e e}$ on them.

The standard deviations are taken so that the condition is satisfied:

$$
\sqrt{\left(M_{\omega i}-M_{\omega j}\right)^{2}+\left(M_{M i}-M_{M j}\right)^{2}} \geq \sqrt{\left(2 \cdot \sigma_{\omega i}-2 \cdot \sigma_{\omega j}\right)^{2}+\left(2 \cdot \sigma_{M i}-2 \cdot \sigma_{M j}\right)^{2}} .
$$

This will ensure that there is no significant mutual influence when adjusting the fuel consumption values for the indicated engine operation modes. The theoretical values of fuel consumption $g_{e t}$ are calculated using (1), (3) and (5) by substituting the values of mathematical expectations $M_{\omega}, M_{M}, M_{N}$ and compared with experimental $g_{e e}$. Next, the difference between the experimental and theoretical values is determined

$$
\Delta_{i}=g_{e e i}-g_{e t i}
$$

The refinement coefficients are calculated for the probability density function of the two-dimensional distribution law for the indicated modes

$$
\tau_{i}=2 \cdot \pi \cdot \Delta_{i} \cdot \sigma_{M i} \cdot \sigma_{\omega i}
$$

This allows to adjust the theoretical values of specific fuel consumption so that they coincide with the available experimental ones at $n$ points using the probability density functions of the two-dimensional normal distribution, the superposition of which forms the surface, and the fuel consumption

$$
f_{g e k}\left(\varepsilon_{\omega}, \varepsilon_{M}, M_{\omega i}, M_{M i}, \sigma_{\omega i}, \sigma_{M i}, \tau_{i},\right)=\sum_{i=1}^{n} \frac{\tau_{i}}{2 \cdot \pi \cdot \sigma_{\omega i} \cdot \sigma_{M i}} \cdot \exp \left(-\frac{1}{2} \cdot\left(\begin{array}{c}
\frac{\left(\varepsilon_{\omega}-M_{\omega i}\right)^{2}}{\sigma_{\omega i}^{2}}+ \\
+\frac{\left(\varepsilon_{M}-M_{M i}\right)^{2}}{\sigma_{M i}^{2}}
\end{array}\right)\right) .
$$

Then the adjusted specific fuel consumption

$$
g_{e k}=g_{n} \cdot k_{N} \cdot k_{\omega}+f_{g e k}\left(\varepsilon_{\omega}, \varepsilon_{M}, M_{\omega i}, M_{M i}, \sigma_{\omega i}, \sigma_{M i}, \tau_{i}\right)
$$

For simulation at partial loads and engine speed operating modes, it is proposed to obtain the full-throttle torque characteristic by approximating the spline in the normalized form $\mathrm{M}_{\text {norm }}$ so that the nominal mode corresponds to the point $(1 ; 1)$

$$
M_{n o r m}=f_{M}\left(\varepsilon_{\omega}\right)
$$

Effective moment $M_{e}$ and engine power $N_{e}$

$$
\begin{gathered}
M_{e}=M_{n} \cdot \varepsilon_{M} \\
N_{e}=M_{e} \cdot \omega=M_{e} \cdot \varepsilon_{\omega} \cdot \omega_{n}=M_{n} \cdot \varepsilon_{M} \cdot \varepsilon_{\omega} \cdot \omega_{n}=N_{n} \cdot \varepsilon_{N},
\end{gathered}
$$

where $M_{n}-$ the rated torque of the engine; $\omega_{n}-$ the angular velocity, which corresponds to the rated speed $n_{n} ; N_{n}$ - the rated engine power; $\varepsilon_{\omega}, \varepsilon_{\mathrm{M}}, \varepsilon_{N}$ - engine relative loading by angular velocity, torque and power, respectively. 
The specific fuel consumption depends on the speed and load conditions of the engine, namely $\varepsilon_{\omega}$ and $\varepsilon_{M}$. Let's change these values $\varepsilon_{\omega} \in\left[\omega_{\text {min }} / \omega_{\text {rated }}, k_{x}\right], \varepsilon_{M} \in\left[\varepsilon_{\mathrm{Mmin}}, k_{\mathrm{M}}\right]$, where $\omega_{\text {min }}-$ the minimum angular velocity of the crankshaft, $\varepsilon_{\mathrm{Mmin}}=(0,3 \ldots 0,4) ; k_{x}$ - coefficient determining the regulatory branch of the full-throttle characteristics, $k_{x}=1.04 \ldots 1.10 ; k_{M}$ - the safety factor of torque. The $\varepsilon_{M}$ value is limited by the normalized engine torque $M_{n o r m}$, which corresponds to the full-throttle characteristic.

To determine the operating modes of a diesel engine, use the differential equation of motion of the shaft with the parameters given to it

$$
J \cdot \dot{\omega}=M_{e}-M_{c}
$$

where $J, M_{c}-$ moment of inertia of the tractor with the unit and the moment of resistance to movement reduced to the engine shaft.

To take into account the possible partial engine load and the dependence of the effective torque on the speed mode and the position of the fuel control, let's introduce the coefficient $\boldsymbol{\varepsilon}_{r}$. This coefficient characterizes the position of the fuel control. Then the relationship of engine performance can be written as follows

$$
\begin{gathered}
\varepsilon_{\omega}=\varepsilon_{r}+\left(M_{\text {norm }}-\varepsilon_{M}\right) \cdot\left(k_{x}-1\right) . \\
M_{e}=\left(M_{\text {norm }}+\frac{\varepsilon_{r}-\varepsilon_{\omega}}{k_{x}-1}\right) \cdot M_{n} .
\end{gathered}
$$

After transformations of equation (13), taking into account (15), let's obtain in relative quantities in the form

$$
J \cdot \dot{\varepsilon}_{\omega} \cdot \omega_{n}=\left(M_{n o r m}+\frac{\varepsilon_{r}-\varepsilon_{\omega}}{k_{x}-1}-\varepsilon_{M}\right) \cdot M_{n} .
$$

The proposed equation (16) differs from the existing ones in that it allows one to realize any regularity in time of a change in the external resistance or $\varepsilon_{M}$ and fuel supply control $\varepsilon_{r}$. The totality of all the above parameters form a multi-parameter engine characteristic, which calculates the instantaneous fuel consumption $\left(G_{\mathrm{M}}, \mathrm{g} / \mathrm{s}\right)$ and fuel consumption $\left(G_{t M}, \mathrm{~g}\right)$ for the time interval $t \in\left[t_{s}, t_{e}\right]$

$$
\begin{gathered}
G_{M}=\frac{g_{e} \cdot \varepsilon_{N} \cdot N_{n}}{3,6 \cdot 10^{6}} ; \\
G_{t M}=\int_{t_{s}}^{t_{e}} \frac{g_{e} \cdot \varepsilon_{N} \cdot N_{n}}{3,6 \cdot 10^{6}} \mathrm{~d} t=\frac{N_{n}}{3,6 \cdot 10^{6}} \cdot \int_{t_{s}}^{t_{e}} g_{e} \cdot \varepsilon_{N} \mathrm{~d} t .
\end{gathered}
$$

Work $(\mathrm{A}, \mathrm{kW} \cdot \mathrm{h})$ and average specific fuel consumption $g_{\text {em }}$ per time $t \in\left[t_{s}, t_{e}\right]$

$$
\begin{gathered}
A=\int_{t_{s}}^{t_{e}} \frac{\varepsilon_{N} \cdot N_{n}}{3600} \mathrm{~d} t=\frac{N_{n}}{3600} \int_{t_{s}}^{t_{e}} \varepsilon_{N} \mathrm{~d} t \\
g_{e m}=\frac{G_{t M}}{A}=\frac{1}{1000} \cdot \int_{t_{s}}^{t_{e}} g_{e} \cdot \varepsilon_{N} \mathrm{~d} t / \int_{t_{s}}^{t_{e}} \varepsilon_{N} \mathrm{~d} t .
\end{gathered}
$$

The mathematical model of the engine characteristic and its specific fuel consumption makes it possible to estimate fuel economy taking into account the tractor's variable pulling load over time. 


\section{The results of modeling the fuel economy of a wheel-type tractor when loading in field} cycles PowerMix

The test report of the Fendt 942 Vario Gen 6 tractor [11] was taken as the initial data for modeling the specific $g_{e}$ and hourly $G_{t}$ fuel consumption and approbation of the proposed mathematical model, the data of which when obtaining the engine characteristics through the power take-off shaft (PTO) are given in Table 2 and in Fig. 1.

Table 2

Engine performance obtained on the tractor power take-off shaft [11]

\begin{tabular}{|c|c|c|c|c|c|}
\hline Mode & $\begin{array}{c}\text { Rotation } \\
\text { frequency, } \text { min }^{-1}\end{array}$ & $\begin{array}{c}\text { Power per GDP, } \\
\text { kW }\end{array}$ & $\begin{array}{c}\text { Equivalent } \\
\text { moment, } \mathrm{Nm}\end{array}$ & $G_{t}, \mathrm{~kg} / \mathrm{h}$ & $g_{e}, g / k W \cdot h$ \\
\hline rated power & 1700 & 277 & 1555 & 58.4 & 211 \\
\hline maximum power & 1700 & 277 & 1555 & 58.4 & 211 \\
\hline maximum torque & 1350 & 257 & 1816 & 52.8 & 206 \\
\hline $1000 \mathrm{rpm}$ РTO & 1604 & 275 & 1637 & 57.5 & 210 \\
\hline \multicolumn{6}{|c|}{ Partial load modes (\# 1-5) according to OECD Code 2 [9] } \\
\hline Full throttle, $80 \%$ of $N_{n}(\# 1)$ & 1742 & 221 & 1214 & 47.8 & 216 \\
\hline $90 \%$ of $n_{n}, 80 \%$ of $N_{n}(\# 2)$ & 1535 & 222 & 1383 & 46.5 & 209 \\
\hline $90 \%$ of $n_{n}, 40 \%$ of $N_{n}(\# 3)$ & 1532 & 111 & 691 & 26.1 & 236 \\
\hline $60 \%$ of $n_{n}, 60 \%$ of $N_{n}(\# 4)$ & 1050 & 166 & 1510 & 34.2 & 206 \\
\hline $60 \%$ of $n_{n}, 40 \%$ of $N_{n}(\# 5)$ & 1021 & 111 & 1038 & 23.6 & 212 \\
\hline
\end{tabular}

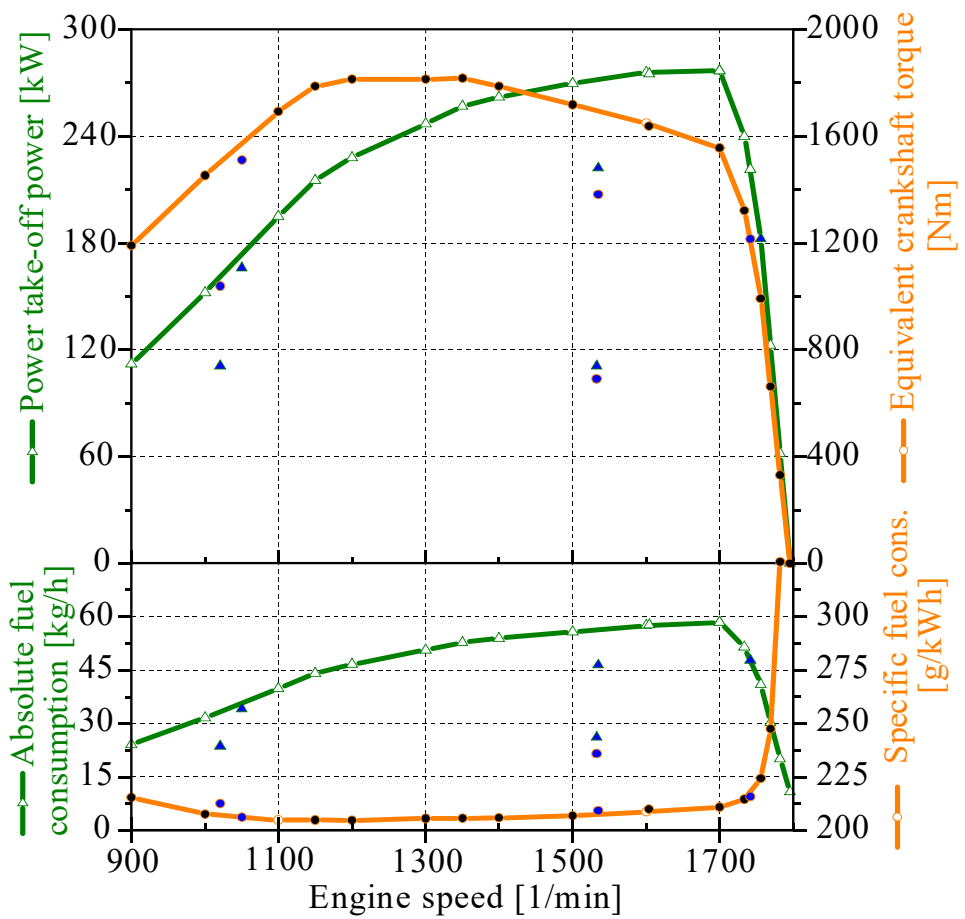

Fig. 1. Engine performance obtained on the tractor power take-off shaft [11]

The results of the approximation of the specific fuel consumption $g_{e}(1)$, adjusted for particular modes (\# 1-5, Table 2) $g_{e k}(10)$ are shown in Fig. 2. 


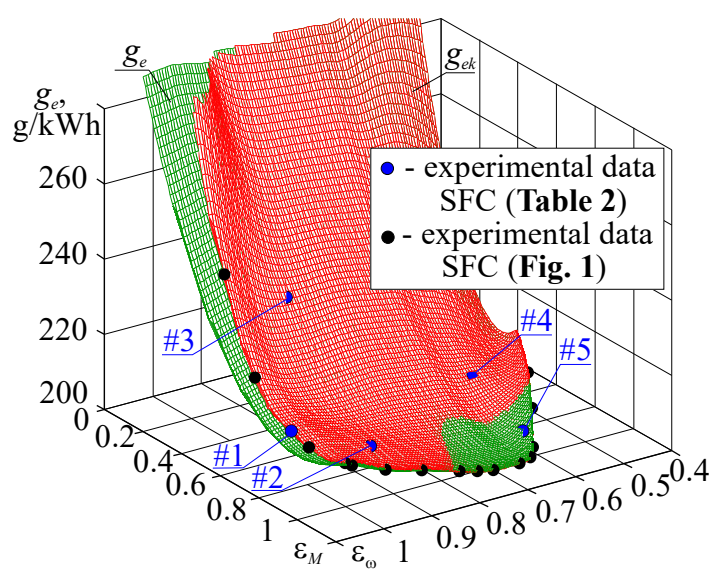

$a$

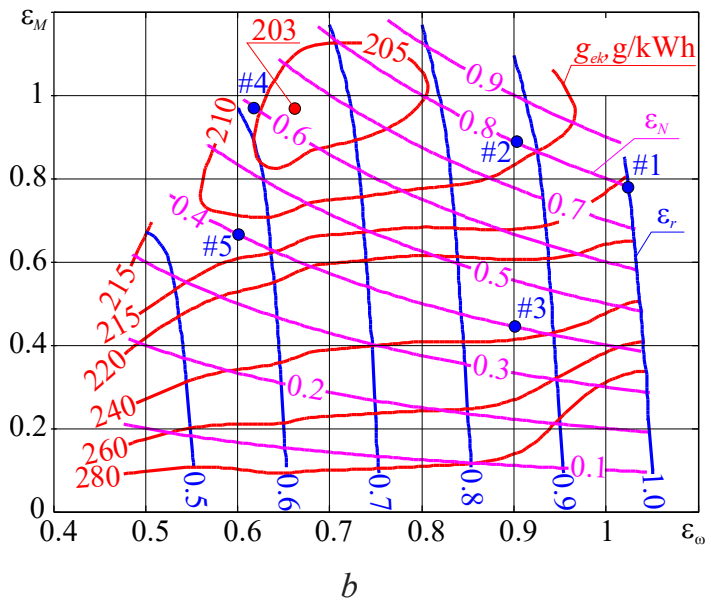

Fig. 2. Specific fuel consumption model of the engine of the Fendt 942 Vario tractor: $a$ - surfaces $g_{e}$ and $g_{e k} ; b$ - contour graphs $g_{e k}, \varepsilon_{N}, \varepsilon_{r}$

A mathematical model of engine characteristics and specific fuel consumption allowed to carry out simulation modeling with variable traction load for PowerMix field cycles, the results of which are given in Table 3.

Table 3

Performance and fuel consumption of the Fendt 942 Vario Gen 6 tractor [11]

\begin{tabular}{|c|c|c|c|c|c|c|}
\hline \multirow{2}{*}{$\begin{array}{l}\text { PowerMix Field Performance and Fuel } \\
\text { Consumption (Experiment/Calculation) }\end{array}$} & \multirow{2}{*}{$\begin{array}{c}\begin{array}{c}\text { Rotation } \\
\text { frequency, }\end{array} \\
\text { min }^{-1}\end{array}$} & \multirow{2}{*}{$\begin{array}{c}\text { Speed } \\
\mathbf{k m} / \mathbf{h}\end{array}$} & \multirow{2}{*}{$\begin{array}{c}\text { Power } \\
k W\end{array}$} & \multicolumn{2}{|c|}{ Fuel consumption $G_{t}$} & \multirow{2}{*}{$\begin{array}{c}\begin{array}{c}\text { Fuel con- } \\
\text { sumption } \mathbf{g}_{\mathrm{e}}\end{array} \\
\mathrm{g} / \mathbf{k W} \cdot \mathbf{h}\end{array}$} \\
\hline & & & & $\mathrm{kg} / \mathrm{h}$ & l/h & \\
\hline 1 & 2 & 3 & 4 & 5 & 6 & 7 \\
\hline \multirow{2}{*}{ Z1P plow $100 \%$} & 1333 & 7.5 & 210 & 51.2 & 61.0 & 244 \\
\hline & 1328 & 6.5 & 202 & 49.4 & 59.0 & 245 \\
\hline \multirow[t]{2}{*}{ Z1P non-compliance percentage, $\%$} & -0.4 & -13.3 & -3.8 & -3.5 & -3.5 & +0.4 \\
\hline & 1503 & 9.4 & 218 & 53.6 & 63.9 & 246 \\
\hline Z1G cultivator $100 \%$ & 1502 & 8.8 & 207 & 53.6 & 63.9 & 249 \\
\hline
\end{tabular}




\begin{tabular}{|c|c|c|c|c|c|c|}
\hline 1 & 2 & 3 & 4 & 5 & 6 & 7 \\
\hline Z1G non-compliance percentage, $\%$ & 0 & -8.5 & -5.1 & -4.1 & -4.1 & +1.2 \\
\hline \multirow{2}{*}{ Z2P plow $60 \%$} & 1143 & 8.6 & 147 & 35.5 & 42.3 & 242 \\
\hline & 1150 & 7.4 & 148 & 36.2 & 43.2 & 245 \\
\hline Z2P non-compliance percentage, $\%$ & +0.6 & -13.9 & +0.7 & +2.0 & +2.0 & +1.2 \\
\hline \multirow{2}{*}{ Z2G cultivator $60 \%$} & 1219 & 11.6 & 167 & 40.6 & 48.4 & 242 \\
\hline & 1224 & 10.6 & 168 & 41.1 & 49.1 & 245 \\
\hline Z2G non-compliance percentage, $\%$ & +0.4 & -8.6 & +0.6 & +1.2 & +1.2 & +1.2 \\
\hline \multirow{2}{*}{ Z3K rotary harrow $100 \%$} & 1391 & 5.5 & 225 & 49.0 & 58.4 & 218 \\
\hline & 1385 & 5.0 & 220 & 48.8 & 58.3 & 222 \\
\hline Z3K non-compliance percentage, $\%$ & -0.4 & -9.1 & -2.2 & -0.4 & -0.4 & +1.8 \\
\hline \multirow{2}{*}{ Z3M mower $100 \%$} & 1383 & 14.0 & 227 & 51.8 & 61.8 & 228 \\
\hline & 1380 & 14.0 & 216 & 49.9 & 59.6 & 231 \\
\hline Z3M non-compliance percentage, $\%$ & -0.2 & 0 & -4.8 & -3.7 & -3.7 & +1.3 \\
\hline \multirow{2}{*}{$\mathrm{Z} 4 \mathrm{~K}$ rotary harrow $70 \%$} & 1132 & 5.6 & 160 & 35.0 & 41.8 & 218 \\
\hline & 1106 & 5.3 & 151 & 33.7 & 40.3 & 224 \\
\hline Z4K non-compliance percentage, $\%$ & -2.3 & -5.4 & -5.6 & -3.7 & -3.7 & +2.8 \\
\hline \multirow{2}{*}{ Z4M mower $70 \%$} & 1110 & 14.2 & 160 & 36.9 & 44.0 & 230 \\
\hline & 1120 & 14.3 & 156 & 36.3 & 43.3 & 233 \\
\hline Z4M non-compliance percentage, $\%$ & +0.9 & +0.7 & -2.5 & -1.6 & -1.6 & +1.3 \\
\hline \multirow{2}{*}{$\mathrm{Z} 5 \mathrm{~K}$ rotary harrow $40 \%$} & 1145 & 6.0 & 95 & 22.8 & 27.2 & 241 \\
\hline & 1132 & 5.7 & 99 & 24.1 & 28.7 & 243 \\
\hline Z5K non-compliance percentage, $\%$ & -1.1 & -5.0 & +4.2 & +5.7 & +5.7 & +0.8 \\
\hline \multirow{2}{*}{$\mathrm{Z} 5 \mathrm{M}$ mower $40 \%$} & 1142 & 15.9 & 100 & 25.4 & 30.3 & 255 \\
\hline & 1143 & 15.3 & 107 & 26.8 & 31.9 & 250 \\
\hline Z5M non-compliance percentage, $\%$ & +0.1 & -3.8 & +7.0 & +5.5 & +5.5 & -2.0 \\
\hline \multirow{2}{*}{ Z6MS manure spreading } & 1256 & 5.7 & 170 & 39.7 & 47.4 & 234 \\
\hline & 1255 & 5.7 & 170 & 39.7 & 47.4 & 233 \\
\hline Z6MS non-compliance percentage, $\%$ & -0.1 & 0 & 0 & 0 & 0 & -0.4 \\
\hline \multirow[b]{2}{*}{ Z7PR baler } & 1261 & 7.6 & 146 & 35.5 & 42.3 & 243 \\
\hline & 1252 & 7.6 & 145 & 33.3 & 39.7 & 230 \\
\hline Z7PR non-compliance percentage, $\%$ & -0.7 & 0 & -0.7 & -6.2 & -6.2 & -5.3 \\
\hline Experiment average fuel consumption & & & & & & 237 \\
\hline Simulated model average fuel consumption & & & & & & 237 \\
\hline
\end{tabular}


The results of the implementation of the mathematical model of specific fuel consumption completely coincide with the experimental data, both on the external speed characteristic (black dots, Fig. 2, a) and on partial modes \# 1-5 (blue dots, Fig. 2, a). After approximating the surface of the specific fuel consumption by $k_{\omega}-$ spline, $k_{N}$ - by a polynomial of the second degree of the largest adjustment, a regime near the regulatory branch of the full-throttle characteristic is required. In this area, there is a significant difference in the surfaces of specific fuel consumption before the correction $g_{e}$ and after the correction $g_{e k}$ (Fig. 2, a).

In general, the proposed method for simulating specific fuel consumption ensures that the calculated data are fully consistent with the available experimental points. Comparison of the results of a simulation of tractor profitability when loading in field cycles PowerMix showed a satisfactory convergence of theoretical and experimental data. The maximum discrepancy between the indicators is: crankshaft speed $(-2.3 \ldots+0.4) \%$; tractor speed $(-13.9 \ldots+0.7) \%$; transmitted power $(-5.6 \ldots+7.0) \%$; hourly fuel consumption $(-6.2 \ldots+5.7) \%$; specific fuel consumption $(-5.3 \ldots+2.8) \%$. The average value of the specific fuel consumption in the PowerMix field cycles, as the main indicator of profitability, coincides according to the results of modeling and experiment.

The difference between the calculation and the experiment is explained by the fact that, given the exact fuel characteristic of the engine, there is no data on the transmission efficiency and its dependence on the transmitted power, power loss on the auxiliary equipment drive. Also, during computer simulation, the selected gear ratio of a continuously variable transmission can significantly affect the result, it provides engine loading with a torque of $\varepsilon_{\mathrm{M}}$. According to the DLG PowerMix test reports, engine load can only be indirectly estimated by hourly fuel consumption.

Since the DLG PowerMix test reports contain information specifically about the transmitted power, they can also be used to determine the tractor's traction performance not on the concrete track, but on the agricultural soil. This is exactly what was done in the framework of this work. Therefore, in almost all test cycles, the actual tractor speed according to the simulation results is lower due to higher slippage of tires on the soil than on a concrete track. This is especially noticeable in energy-intensive operations of Z1P, Z1G, Z2P, Z2G, Z3K cycles.

\section{Discussion of the results of modeling the fuel economy of a wheel-type tractor when loading in field cycles PowerMix}

The obtained dependences in the materials of this article are the first step in an in-depth analysis of the traction efficiency of existing tractors and justification of the design indicators of the tractors.

Testing the above methodology for approximating the fuel characteristics together with a mathematical model of the full-throttle and partial speed characteristics of a diesel engine showed that the calculation results coincide with the experimental points. If there are more experimental points according to the mathematical model, the calculation results can be further refined on the modes remote from the full-throttle characteristics and experimental modes \# 1-5 (Table 2, Fig. 2).

The satisfactory convergence of experimental data and theoretical calculations on PowerMix cycles when simulating tractor movement on the ground allows to state the advisability of using the declared mathematical apparatus.

The peculiarity of the study is that, unlike the existing ones, when determining the fuel economy of a wheel-type tractor, the characteristic of the fuel consumption of the engine is approximated with high accuracy by the above method. This allows to move on to the use of DLG PowerMix test field cycles that simulate the dynamic load on the tractor when simulating movement on agricultural soil instead of a concrete track. 
The use of the proposed model may be limited in the absence or insufficient amount of experimental data on fuel consumption by the tractor engine in partial modes. But the model can be adapted to a different amount of experimental data, which, of course, will affect its accuracy.

The prospect of further research in the outlined direction is the determination of technical and economic indicators of tractors and analysis of the structure of power losses during simulation tests on PowerMix field cycles. At the same time, it is planned to pay special attention to the load conditions of tractor tires, the regulation of the necessary internal pressure in them, and the environmental aspect of the tire-soil interaction.

\section{Conclusions}

The proposed method for approximating the specific fuel consumption of a diesel engine is based on experimental test data on a full-throttle characteristic and on partial engine operation modes. It is proposed to approximate the full-throttle characteristic of specific fuel consumption by a spline, and for partial loads, to carry out the approximation by a polynomial of the second degree using the data of fuel consumption at modes at a speed of 0.9 of the nominal. These modes according to the OECD Code 2 protocol [11] are contained in the test results of tractors [7]. The surface area of specific fuel consumption can be approximated using experimental data on fuel consumption in all partial modes, as well as on the regulatory branch of the full-throttle characteristic. Such a refinement can be carried out using the probability density function of the two-dimensional normal distribution of random variables, which allows to obtain complete agreement between the calculation and the experiment at the available experimental points.

The mathematical model of the characteristics of the diesel engine and the approximate specific fuel consumption is tested on the example of the Fendt 942 Vario Gen 6 tractor as part of a complex mathematical model for determining the traction dynamics of a wheel-type tractor. The results of simulation showed that they coincide with the experimental data obtained with PowerMix field cycles, with an error that does not exceed $2.3 \%$ for the crankshaft rotational speed, $13.9 \%$ of the actual tractor speed, $7.0 \%$ of the transmitted power, and hourly fuel consumption $6.2 \%$, specific fuel consumption of $5.3 \%$. The difference between the calculation and the experiment is explained by the lack of data on the efficiency of a continuously variable transmission, its dependence on the transmitted power and losses on the auxiliary equipment drive.

The biggest difference in the actual speed is due to simulation of PowerMix cycles on the soil, as well as a higher slipping of tires compared to movement on a concrete track.

In the following, a mathematical model can be used to analyze the structure of tractor power losses and the environmental aspect of the interaction of the tire with the ground during traction and dynamic simulation tests of tractors.

\section{References}

[1] Grisso, R. (2014). Predicting Tractor Diesel Fuel Consumption. Virginia Cooperative Extension. Publication 442-073. Available at: https://www.pubs.ext.vt.edu/content/dam/pubs_ext_vt_edu/442/442-073/BSE-328.pdf

[2] Grisso, R. D., Kocher, M. F., Vaughan, D. H. (2004). Predicting tractor fuel consumption. Applied Engineering in Agriculture, 20 (5), 553-561. doi: https://doi.org/10.13031/2013.17455

[3] Kocher, M. F., Smith, B. J., Hoy, R. M., Woldstad, J. C., Pitla, S. K. (2017). Fuel Consumption Models for Tractor Test Reports. Transactions of the ASABE, 60 (3), 693-701. doi: https://doi.org/10.13031/trans.12121

[4] Grisso, R. (2014). "Gear Up and Throttle Down” to Save Fuel. Virginia Cooperative Extension. Publication 442-450. Available at: https://www.pubs.ext.vt.edu/content/dam/pubs_ext_vt_edu/442/442-450/BSE-326.pdf

[5] Coffman, B. A., Kocher, M. F., Adamchuk, V. I., Hoy, R. M., Blankenship, E. E. (2010). Testing Fuel Efficiency of a Tractor with a Continuously Variable Transmission. Applied Engineering in Agriculture, 26 (1), 31-36. doi: https://doi.org/ 10.13031/2013.29468 
[6] Howard, C. N., Kocher, M. F., Hoy, R. M., Blankenship, E. E. (2013). Testing the Fuel Efficiency of Tractors with Continuously Variable and Standard Geared Transmissions. Transactions of the ASABE, 56 (3), 869-879. doi: https://doi.org/10.13031/ trans.56.10222

[7] DLG Test Report database. Available at: https://www.dlg.org/en/agriculture/tests/query-for-test-reports

[8] Nebraska tractor test laboratory. Test reports. Available at: https://ractortestlab.unl.edu/testreports

[9] OECD standard code for the official testing of agricultural and forestry tractor performance. CODE 2. Available at: http:// www.oecd.org/agriculture/tractors/codes/02-oecd-tractor-codes-code-02.pdf

[10] Friso, D. (2014). Brake thermal efficiency and BSFC of diesel engines: Mathematical modeling and comparison between diesel oil and biodiesel fueling. Applied Mathematical Sciences, 8, 6515-6528. doi: https://doi.org/10.12988/ams.2014.46444

[11] Fendt 942 Vario Gen6. DLG Test Report 7042. Available at: https://pruefberichte.dlg.org/filestorage/PowerMix_Datenblatt_ FENDT_942_Gen6_EN_V2.pdf 